\title{
Homozygosity mapping and whole-genome sequencing reveals a deep intronic PROM1 mutation causing cone-rod dystrophy by pseudoexon activation
}

\author{
Anja K Mayer ${ }^{1}$, Klaus Rohrschneider ${ }^{2}$, Tim M Strom ${ }^{3}$, Nicola Glöckle ${ }^{4}$, Susanne Kohl ${ }^{1}$, Bernd Wissinger ${ }^{1}$ \\ and Nicole Weisschuh ${ }^{\star 1}$
}

Several genes have been implicated in the autosomal recessive form of cone-rod dystrophy (CRD), but the majority of cases remain unsolved. We identified a homozygous interval comprising two known genes associated with the autosomal recessive form of CRD, namely RAB28 and PROM1, in a consanguineous family with clinical evidence of CRD. Both genes proved to be mutation negative upon sequencing of exons and canonical splice sites but whole-genome sequencing revealed a private variant located deep in intron 18 of PROM1. In silico and functional analyses of this variant using minigenes as splicing reporters revealed the integration of a pseudoexon in the mutant transcript, thereby leading to a premature termination codon and presumably resulting in a functional null allele. This is the first report of a deep intronic variant that acts as a splicing mutation in PROM1. The detection of such variants escapes the exon-focused techniques typically used in genetic analyses. Sequencing the entire genomic regions of known disease genes might identify more causal mutations in the autosomal recessive form of CRD.

European Journal of Human Genetics (2016) 24, 459-462; doi:10.1038/ejhg.2015.144; published online 8 July 2015

\section{INTRODUCTION}

Cone-rod dystrophy (CRD; MIM 120970) has an estimated prevalence of 1:30000 to 1:40000 and is characterized by primary loss of cone function and subsequent or simultaneous loss of rod vision. ${ }^{1}$ Key symptoms are poor visual acuity, dyschromatopsia and photophobia.

CRDs are genetically heterogeneous and can show different modes of Mendelian inheritance, with autosomal recessive (ar) traits being more common than autosomal dominant and X-linked forms. Currently, 17 genes have been implicated in arCRD, with ABCA4 (MIM 601691) being the major contributing gene, whereas each of the other known genes, among them PROM1 (MIM 612657), account for only a minor fraction of cases. ${ }^{2}$ About $75 \%$ of arCRD cases apparently do not harbor mutations in these known genes. ${ }^{2}$

Here we report on a consanguineous family with CRD. The two affected siblings were diagnosed with CRD in the second decade of life, with rapidly deteriorating visual acuities. As the two affected siblings were born out of a first-cousin marriage, we expected the diseasecausing mutation to be located in a homozygous interval. ${ }^{3}$ To that end, genetic analyses started with the identification of these, followed by a panel-based next-generation sequencing approach and finally whole-genome sequencing.

\section{PATIENTS AND METHODS}

Members of the family originated from southwest Germany and were recruited in accordance with the principles of the Declaration of
Helsinki, with informed consent and ethical approval. DNA was isolated from venous blood samples using conventional techniques. Using Affymetrix CytoScan HD Arrays (Affymetrix, Santa Clara, CA, USA), regions of homozygosity were calculated with the online tool Homozygosity Mapper (http://www.homozygositymapper.org/). ${ }^{4}$ Panel-based sequencing of 105 retinal degenerationassociated genes and whole-genome sequencing, respectively, were performed as described before. ${ }^{5,6}$ In-house automated data analysis pipeline and variant interpretation tools were used for variant calling.

A 2.7-kb fragment comprising PROM1 exon 18 with $239 \mathrm{bp}$ of upstream sequence, intron 18 and exon 19 with $367 \mathrm{bp}$ of downstream sequence was amplified using a proofreading polymerase and genomic DNA from family member I:2 (see Figure 1) in order to co-amplify both the normal and the mutant allele. Cloning into the exon-trapping vector pSPL3, transfection of human embryonic kidney (HEK) 293T cells, RNA isolation and cDNA synthesis were performed as described previously. ${ }^{7}$ Transfection and downstream analyses of murine $661 \mathrm{~W}$ photoreceptor-derived cells were performed likewise.

The identified variant was submitted to ClinVar (www.ncbi.nlm. nih.gov/clinvar/) with accession code SCV000222908.

\section{RESULTS}

Homozygosity mapping of the two affected siblings revealed a 7-Mb shared homozygous interval on chromosome 4 (rs12512447-rs7699941). The interval comprises 21 HGNC-approved protein-coding genes,

\footnotetext{
${ }^{1}$ Molecular Genetics Laboratory, Centre for Ophthalmology, Institute for Ophthalmic Research, University of Tuebingen, Tuebingen, Germany; ${ }^{2}$ Department of Ophthalmology, University of Heidelberg, Heidelberg, Germany; ${ }^{3}$ Institute of Human Genetics, Helmholtz Zentrum Muenchen, Neuherberg, Germany; ${ }^{4}$ CeGaT GmbH, Tuebingen, Germany ${ }^{*}$ Correspondence: Dr N Weisschuh, Molecular Genetics Laboratory, Centre for Ophthalmology, Institute for Ophthalmic Research, University of Tuebingen, Roentgenweg 11, D-72076 Tuebingen, Germany. Tel: +49 7071 2987618; Fax: +49 7071 295725; E-mail: nicole.weisschuh@uni-tuebingen.de Received 18 March 2015; revised 19 May 2015; accepted 26 May 2015; published online 8 July 2015
} 


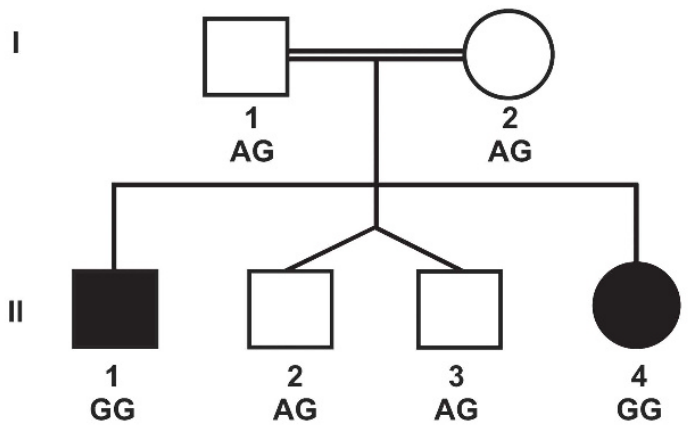

b

wild type

NNSPLICE [0-1] HSF [0-100]

mutant

NNSPLICE [0-1] HSF [0-100]

Figure 1 Pedigree of the CRD family and in silico splicing predictions for the wild-type and mutant genomic sequences of PROM1. (a) Pedigree showing segregation of the PROM1 intronic variant c.2077-521A $>G$. (b) Splice-site prediction for the wild-type (top) and the mutant allele (bottom). The position of the c.2077-521A $>$ G variant is indicated by the vertical dotted line. Donor site scores from NNSPLICE and HSF are displayed as green vertical bars. The prediction score is provided beside each bar.

among them two genes were known to be mutated in arCRD, namely RAB28 and PROM1. However, subsequent Sanger sequencing for $R A B 28$ and targeted next-generation sequencing for PROM1 ruled out variants that may affect function in the exons and the canonical splice sites of the two genes. The targeted next-generation sequencing generated sequence data for additional 104 retinal degenerationassociated genes, ${ }^{5}$ but no putative disease-causing variants were detected in these. Subsequently, we performed whole-genome sequencing of both the affected siblings, generating 159 and $179 \mathrm{~Gb}$ of sequence with an average read depth of 44 and 50, respectively. We found no rare and potentially disease-causing exonic variants compatible with a model of autosomal recessive inheritance and shared by both siblings. In a next step, raw read data of the genomic regions of RAB28 and PROM1 were manually checked with the Integrative Genomics Viewer. ${ }^{8}$ In comparison with the hg19 human reference sequence, we detected 18 singlenucleotide variants in $R A B 28$ and 196 in PROM1, which were shared by both siblings. After filtering out annotated SNPs (dbSNP 137 and 1000 Genomes), only one private variant remained that was located deep in intron 18 of PROM1 (c.2077-521A $>$ G) and segregated with the disease in the expected autosomal recessive mode (Figure 1a). The variant designation is based on the NCBI reference sequence for PROM1 transcript NM_006017.2 and the genomic reference sequence NG_011696.1. The exon numbering is based on NG_011696.1. Splice-site predictions using the online tools Human Splicing Finder (HSF; http://www.umd.be/HSF/) and NNSPLICE (http://www.fruitfly.org/seq_tools/splice.html) showed significantly increased $5^{\prime}$ donor splice-site scores for the sequence surrounding the variant (NNSPLICE 0.99 out of 1; HSF 85.4 out of 100; compared with zero for the wild-type sequence in both prediction programs; see Figure 1b).

To test our hypothesis that this deep intronic variant acts as a splicing mutation, cDNA analyses were performed. Owing to the lack of PROM1 expression in accessible tissues, we made use of a heterologous splicing assay in HEK 293T cells and in murine $661 \mathrm{~W}$ photoreceptor-derived cells to test mutant and wild-type PROM1 minigene constructs in direct comparison. RT-PCR of wild-type RNA resulted in a single product of the expected size (Figure 2a), and bidirectional Sanger sequencing confirmed correct splicing of exons 18 and 19 (Figure 2b). In contrast, RT-PCR of mutant RNA revealed a single transcript larger than the expected correctly spliced product (Figure 2a). Direct sequencing revealed an insertion of a 155-bp cryptic exon spliced between exons 18 and 19 (Figure $2 \mathrm{~b}$ ). The aberrant transcript would lead, if translated, to an insertion of 22 novel amino acids followed by a premature termination codon (Figure 2c). This would result in a truncated protein short of 173 amino acids at the $\mathrm{C}$-terminus and thereby lacking the last of five transmembrane domains. However, it is more likely that the mutant transcript undergoes nonsense-mediated mRNA decay. ${ }^{9}$

\section{DISCUSSION}

The Human Gene Mutation Database (http://www.biobase-international.com/product/hgmd) lists only five PROM1 mutations in arCRD that all lead to functional null alleles: two nonsense mutations, ${ }^{5,10}$ two mutations at canonical splice sites ${ }^{11,12}$ and one single-nucleotide duplication. $^{13,14}$ To the best of our knowledge, this is the first report of a deep intronic variant in PROM1 presumably leading to a functional null allele.

It is important to note that the disease-causing mutation in our family would not have been identified using conventional approaches like Sanger sequencing of coding exons or by using exome sequencing. Genomic analyses for other genes involved in retinal degeneration like $A B C A 4,{ }^{15-17} U S H 2 A,{ }^{18,19} C E P 290^{20}$ and OFD $1^{21}$ have shown that a probably underestimated number of patients harbor deep intronic variants that interfere with splicing. Although the majority of deep intronic variants that act as splicing mutations to date have been identified through the presence of aberrant mRNA transcripts, this approach is only feasible for those genes that are expressed in accessible tissues (ie, lymphocytes, keratinocytes and fibroblasts). Moreover, this approach might be hampered by the degradation of mutant transcripts through nonsense-mediated mRNA decay. These limitations can be overcome by whole-genome sequencing and the use of heterologous splicing assays. Our findings substantiate the necessity of the analysis of regions outside of the coding exons, especially in 


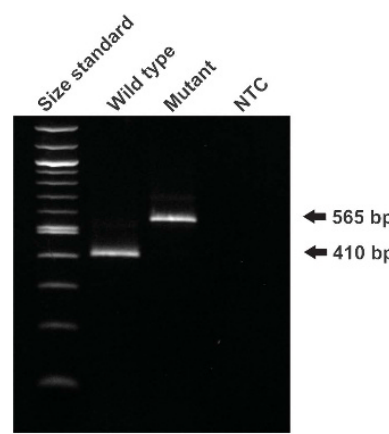

\begin{tabular}{|l|l|l|l|l|}
\hline pSPL3 & exon 18 & cryptic & exon 19 & pSPL3 \\
\hline
\end{tabular}

\begin{tabular}{|l|l|l|l|}
\hline pSPL3 & exon 18 & exon 19 & pSPL3
\end{tabular}

b

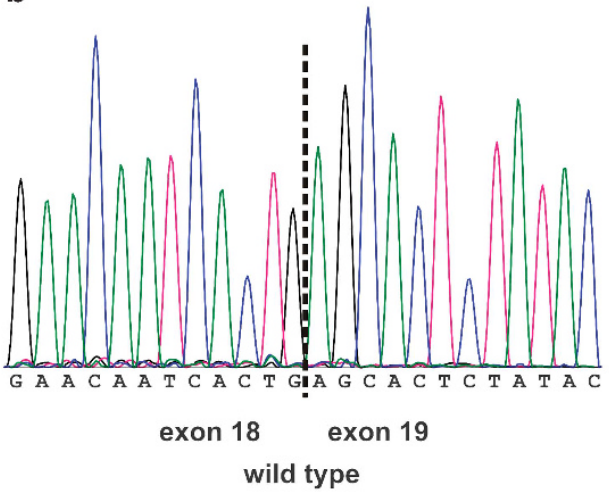

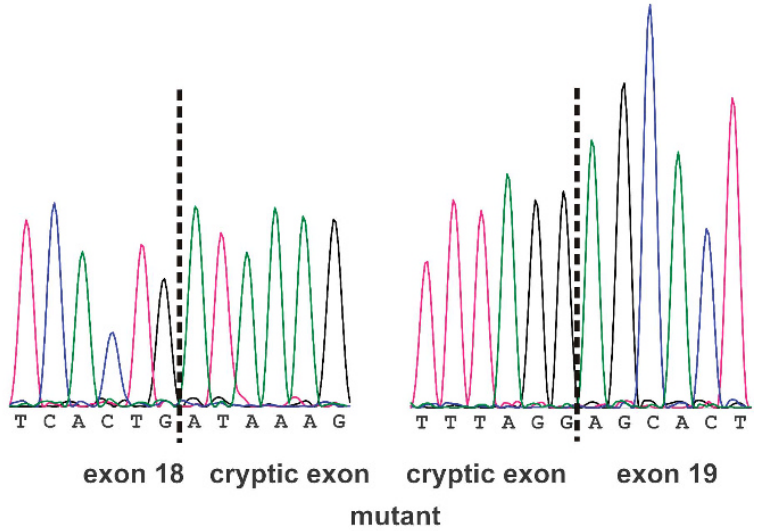

mutant

C

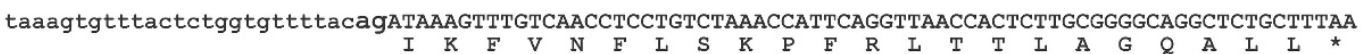

\section{ATAATGCCTTCCACTTTGATCAAACCTAATTCAAAGGGATTCTTGGATGCCTCACATGTGATTTGCTGTTCTTCACAGCTTTAGGgtatgtat}

Figure 2 In vitro splicing assay. As no difference in splicing effect was observed between the two cell lines, only results from HEK 293T cells are shown. (a) RT-PCR revealed an aberrant, larger transcript in HEK 293T cells transfected with the mutant construct compared with the wild-type construct. NTC, nontemplate control. Size standard: NEB 100 bp ladder. Schemes of the amplified products are presented on the right of the agarose gel. White boxes represent PSPL3 exons and gray boxes PROM1 exons. (b) Sequence analysis showing that the aberrant larger transcript is due to the insertion of a cryptic exon of 155 bp between exons 18 and 19. (c) Consequence of the c.2077-521A>G variant (underlined). The cryptic exon (in capital letters) translates into 22 novel amino acids followed by a premature stop codon (protein translation is shown below DNA sequence in one letter amino-acid code).

families with multiple affecteds and evidence of linkage to known disease-gene loci.

\section{CONFLICT OF INTEREST}

The authors declare no conflict of interest.

\section{ACKNOWLEDGEMENTS}

This study was funded by two grants (01GM1108A and 01GM1105A) from the German Federal Ministry for Research and Education (BMBF). We would like to thank Muayyad R Al-Ubaidi for providing us with the $661 \mathrm{~W}$ cell line.

1 Hamel CP: Cone rod dystrophies. Orphanet J Rare Dis 2007; 2: 7

2 Roosing S, Thiadens AA, Hoyng CB, Klaver CC, den Hollander Al, Cremers FP: Causes and consequences of inherited cone disorders. Prog Retin Eye Res 2014; 42: $1-26$.

3 Woods CG, Cox J, Springell K et al: Quantification of homozygosity in consanguineous individuals with autosomal recessive disease. Am J Hum Genet 2006; 78: 889-896.

4 Seelow D, Schuelke M, Hildebrandt F, Nürnberg P: HomozygosityMapper - an interactive approach to homozygosity mapping. Nucleic Acids Res 2009; 37: W593-W599.

5 Glöckle N, Kohl S, Mohr J et al: Panel-based next generation sequencing as a reliable and efficient technique to detect mutations in unselected patients with retinal dystrophies. Eur J Hum Genet 2014; 22: 99-104.
6 Wieczorek D, Newman WG, Wieland T et al: Compound heterozygosity of low-frequency promoter deletions and rare loss-of-function mutations in TXNL4A causes Burn-McKeown syndrome. Am J Hum Genet 2014; 95: 698-707.

7 Weisschuh N, Wissinger B, Gramer E: A splice site mutation in the PAX6 gene which induces exon skipping causes autosomal dominant inherited aniridia. Mol Vis 2012; 18: 751-757.

8 Robinson JT, Thorvaldsdóttir $\mathrm{H}$, Winckler $\mathrm{W}$ et al: Integrative genomics viewer. Nat Biotechnol 2011; 29: 24-26.

9 Frischmeyer PA, Dietz HC: Nonsense-mediated mRNA decay in health and disease. Hum Mol Genet 1999; 8: 1893-1900.

10 Song J, Smaoui N, Ayyagari R et al: High-throughput retina-array for screening 93 genes involved in inherited retinal dystrophy. Invest Ophthalmol Vis Sci 2011; 52: 9053-9060.

11 Littink KW, Koenekoop RK, van den Born LI et al: Homozygosity mapping in patients with cone-rod dystrophy: novel mutations and clinical characterizations. Invest Ophthalmol Vis Sci 2010; 51: 5943-5951.

12 Zhang X, Ge X, Shi W et al: Molecular diagnosis of putative Stargardt disease by capture next generation sequencing. PLoS One 2014; 9: e95528.

13 Pras E, Abu A, Rotenstreich $Y$ et al: Cone-rod dystrophy and a frameshift mutation in the PROM1 gene. Mol Vis 2009; 15: 1709-1716.

14 Wang $\mathrm{F}$, Wang $\mathrm{H}$, Tuan $\mathrm{HF}$ et al: Next generation sequencing-based molecular diagnosis of retinitis pigmentosa: identification of a novel genotypephenotype correlation and clinical refinements. Hum Genet 2014; 133: 331-345.

15 Braun TA, Mullins RF, Wagner $\mathrm{AH}$ et al: Non-exomic and synonymous variants in ABCA4 are an important cause of Stargardt disease. Hum Mol Genet 2013; 22 5136-5145.

16 Bauwens M, De Zaeytijd J, Weisschuh N et al: An augmented ABCA4 screen targeting non-coding regions reveals a deep intronic founder variant in Belgian Stargardt patients. Hum Mutat 2015; 36: 39-42. 
17 Bax NM, Sangermano R, Roosing S et al: Heterozygous deep-intronic variants and deletions in ABCA4 in persons with retinal dystrophies and one exonic ABCA4 variant. Hum Mutat 2015; 36: 43-47.

18 Vaché $\mathrm{C}$, Besnard T, le Berre $\mathrm{P}$ et al: Usher syndrome type 2 caused by activation of an USH2A pseudoexon: implications for diagnosis and therapy. Hum Mutat 2012; 33 : 104-108.

19 Steele-Stallard HB, Le Quesne Stabej P, Lenassi E et al: Screening for duplications, deletions and a common intronic mutation detects $35 \%$ of second mutations in patients with USH2A monoallelic mutations on Sanger sequencing. Orphanet J Rare Dis 2013; 8: 122.

20 den Hollander Al, Koenekoop RK, Yzer S et al: Mutations in the CEP290 (NPHP6) gene are a frequent cause of Leber congenital amaurosis. Am J Hum Genet 2006; 79: 556-561.

21 Webb TR, Parfitt DA, Gardner JC et al: Deep intronic mutation in OFD1, identified by targeted genomic next-generation sequencing, causes a severe form of X-linked retinitis pigmentosa (RP23). Hum Mol Genet 2012; 21: 3647-3654. 